\title{
Unusual localisation of a ventricular septal defect following blunt chest trauma
}

\author{
C Pierli, A Iadanza, A Del Pasqua, G Sinicropi
}

\begin{abstract}
A 64 year old man presented with a traumatic ventricular septal defect following blunt chest trauma 40 years before. Echocardiography and left ventriculography were helpful in locating the unusual septal defect, which was subpulmonary. The shunt was small, but the anomalous chronic overload led to right ventricular failure. The surgical correction was thus too late to improve right ventricular function.

(Heart 2001;86:e6)
\end{abstract}

Keywords: ventricular septal defect; blunt chest trauma; heart failure

Blunt thoracic trauma may cause cardiac rupture in any of the heart chambers or in septal or valvar structures. Interventricular septal rupture, especially when isolated, is a very rare event which has only occasionally been reported. ${ }^{1-7}$ We describe a case in which the site of the defect was particularly unusual and to our knowledge has never previously been described.

\section{Case report}

A 64 year old man was admitted to our department with clinical findings of mild effort dyspnoea, fatigue, syncope, palpitations, and dizziness. He had suffered blunt thoracic trauma caused by the explosion of a land mine about 40 years before, when his sternum was fractured by a piece of shrapnel from the mine. Two months after this trauma, a harsh holosystolic murmur was heard during a follow up examination. Medical examinations before the trauma had revealed no cardiac abnormality and the auscultatory findings had been normal. A small ventricular septal defect was suspected, but owing to the absence of symptoms he did not undergo any further examination. His clinical condition remained satisfactory until the age of 54 years, when he started to experience effort dyspnoea. He underwent cardiac catheterisation which revealed a ventricular septal defect with a left to right shunt (pulmonary to systemic ratio $1.7: 1$ ). On the basis of these data the patient was discharged and treated with digoxin and diuretics.

He returned nine years later suffering from mild effort dyspnoea and palpitations. A harsh holosystolic murmur over the precordium was present and there was right bundle branch block and anterior left bundle branch hemiblock on ECG. Chest $x$ rays showed cardiomegaly with increased pulmonary blood flow. The echocardiogram revealed good left ventricular function, a dilated right ventricle with hypokinetic walls and depressed ventricular function, and a dilated pulmonary trunk with pulmonary regurgitation. The ventricular septal defect seemed to be infundibular and subpulmonary, with two separate jets, the first directed towards the pulmonary valve and the other towards the right ventricular inflow tract. This communication was present at the apex of a bulge in the ventricular septum (fig 1 ).

Repeat cardiac catheterisation data revealed right atrial pressure of $10 \mathrm{~mm} \mathrm{Hg}$, right ventricular pressure of $45 / 10 \mathrm{~mm} \mathrm{Hg}$, pulmonary artery pressure of $40 / 15 \mathrm{~mm} \mathrm{Hg}$, and pulmonary capillary wedge pressure of $14 \mathrm{~mm} \mathrm{Hg}$. The oxygen saturation data showed a pronounced oxygen saturation increment at the ventricular level (superior vena cava $59 \%$, right atrium $60 \%$, right ventricle $71 \%$, right pulmonary artery $75 \%$, right femoral artery $94 \%$ ). The pulmonary to systemic flow ratio was 1.8:1.

Left ventricular angiography confirmed the unusual subpulmonary (infundibular) ventricular septal defect localisation and showed a dilated hypokinetic right ventricle (fig 2). Coronary angiography showed no coronary artery disease.

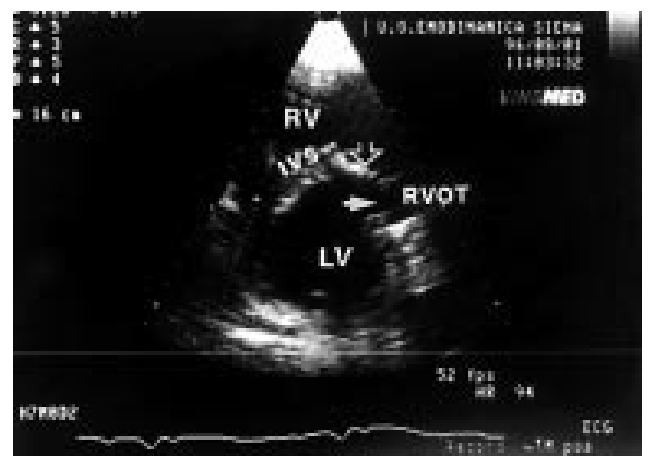

Figure 1 Short axis cross sectional echocardiogram showing the bulge (open arrow) of the muscular outlet septum with a ventricular septal defect (arrow). IVS, interventricular septum; $L V$, left ventricle; $R V$, right ventricle; RVOT, right ventricular outflow tract. 


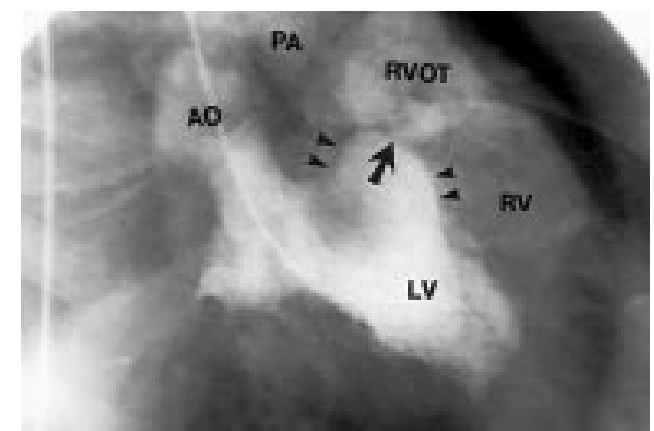

Figure 2 Selective left ventricular angiogram in right anterior oblique view showing a bulge (arrowheads) of the outlet septum with a subpulmonary ventricular septal defect (arrow). The contrast medium passes from the left ventricle to the dilated right ventricular outflow tract. AO, aorta; $L V$, left ventricle; $P A$, pulmonary artery; $R V$, right ventricle; RVOT, right ventricular outflow tract.

Under cardiopulmonary bypass and hypothermic cardioplegic arrest, the ventricular septal defect was exposed through the right atrium and the tricuspid valve. This type of defect is usually exposed through the pulmonary artery in paediatric patients with congenital heart disease, but the surgeon decided on a different approach because of the considerable size of the annulus and the right ventricle. The diameter of the septal defect was approximately $1 \mathrm{~cm}$, with a fibrous band which crossed it horizontally. The site was located at the apex of a bulging muscular infundibular septum, which appeared to be globally irregular and fibrous, as if an inflammatory process had occurred there in the past. The defect was repaired with two Ticron 4-0 stitches mounted on Teflon pledgets reinforced with four interrupted single stitches.

The postoperative course was initially complicated by right ventricular failure and several threatening arrhythmias. However, the patient was discharged on the 13th postoperative day on amiodarone, digoxin, frusemide (furosemide), and captopril.

A postoperative echocardiogram showed a good repair with no residual shunt, but there was mild tricuspid regurgitation with a systolic pulmonary arterial pressure of $30 \mathrm{~mm} \mathrm{Hg}$. The right ventricle remained dilated and hypokinetic. After some months of reasonably good health the patient presented again with right ventricular failure. He had persistent atrial flutter and an advanced grade of atrioventricular block, threatening further arrhythmias. An automatic implantable cardioverterdefibrillator (ICD) was therefore installed and amiodarone treatment continued.

\section{Discussion}

Small post-traumatic ventricular septal defects may cause no symptoms over a long period of time. ${ }^{12}$ A conservative approach is usually indicated for small shunts, even though there is a risk of endocarditis. It is not rare for there to be spontaneous healing or partial closure of the defect, without the development of pulmonary hypertension or right ventricular failure, ${ }^{489}$ but when symptoms occur surgical repair is mandatory.
A ventricular septal perforation located in the infundibular subpulmonary region has never to our knowledge been reported before. The most common site of rupture is believed to be the muscular part of the septum, ${ }^{3}$ and it is more likely to be at the apex than elsewhere. In our patient, however, the perforation was in the subpulmonary region.

The mechanism of rupture is not known for certain. ${ }^{3}$ Compression between the spine and the sternum during direct trauma, a rapid pressure increase during the early systole or late diastole when the valves are closed and the ventricles and septa are in a state of high tension, or acute tearing or contusion with subsequent necrosis and late perforation have all been suggested. ${ }^{7}$ The aneurysmal appearance of the interventricular septum of our case suggests bursting of the ventricle because of a sudden increase in pressure. However, it is not clear whether the defect developed immediately after the trauma or whether it was delayed, which would be the case if was caused by necrosis and subsequent perforation. Because of its proximity to the sternum, the right ventricle is vulnerable to cardiac contusion, and in blunt chest trauma it is often found to be hypokinetic. ${ }^{10}$ This may explain the hypokinetic right ventricular walls that we found on echocardiography in our patient.

In our case the particular site of the defect combined with the abnormal direction of the shunts (directed towards the right ventricular inflow and outflow tracts) led to severe dilatation of the pulmonary trunk and right ventricular outflow tract. This appearance is more typical of pretricuspid shunts than of ventricular septal defects. The chronic overload worsened the function of the probably already damaged right ventricle.

More rigorous post-trauma follow up of this patient would have resulted in earlier surgical treatment of the defect, avoiding the further dilatation of the right ventricle and worsening of right ventricular function that occurred.

1 Evora P, Ribeiro P, Bresil J, et al. Late surgical repair of ventricular septal defect due to non-penetrating chest trauma: review and report of two contrasting cases. $\mathcal{f}$ Trauma review and report

2 Pezarda FA, Mc Dowell JW, Cohen EM, et al. Traumatic ventricular septal defect: sequential hemodynamic observation. N Engl f Med 1974;291:892-5.

3 Parmeley LF, Manion WC, Mattingly TW. Non-penetrating traumatic injury of the heart. Circulation 1958;18:371-96.

4 Rosenthal A, Paris LE, Nadas AS. Isolated interventricular septal defect due to non-penetrating trauma: report of a case with spontaneous healing. $N$ Engl f Med 1970;283: 338-41

5 Rubenstein P, Levinson OC. Acquired interventricular septal defect due to myocardial infarction and non-penetrating trauma to the chest. Am $\mathcal{F}$ Cardiol 1961;7:277-82.

6 Pickard LR, Mattox KL, Beall AC. Ventricular septal defect from blunt chest injury. F Trauma 1980;20:329-31.

7 Rotman M, Peter RM, Searly WC, et al. Traumatic ventricular septal defect secondary to non penetrating chest trauma. Am f Med 1970;48:127-31.

8 Glancy DL, Robert WC. Complete spontaneous closure of VSD: necropsy study of five subjects. Am $\mathcal{F} \mathrm{Med}$ 1967;43:846-53.

9 Walker WJ. Spontaneous closure of traumatic ventricular septal defect. Am f Cardiol 1965;15:263-6.

10 Garcìa-Fernàndez MA, Lòpez-Pérez JM, Castellano NP, et al. Role of transesophageal echocardiography in the assessment of patients with blunt chest trauma: correlation of echocardiographic findings with the electrocardiogram and creatine kinase monoclonal antibody measurements. $\mathrm{Am}$ Heart f 1998;135:477-81. 